\title{
Perspectives on illness-related stigma and electronically sharing psychiatric health information by people with Multiple Sclerosis.
}

Eileen $\mathrm{Yu}$ Johns Hopkins University School of Medicine

Alexis Adams-Clark

University of Oregon

Alison Riehm

Duke University

Caroline Franke

Cleveland Clinic

\section{Ryoko Susukida}

Johns Hopkins University Bloomberg School of Public Health

Melissa Pinto

University of California Irvine

Steven Arenberg

Johns Hopkins Medicine

Dominique Tosi

MedStar Health

Abbey Hughes

Johns Hopkins Medicine

Amanda Montague

Multiple Sclerosis Association of America

Anupama Kumar

Johns Hopkins University School of Medicine

Kay Jamison

Johns Hopkins Medicine

Adam Kaplin ( $\square$ akaplin@jhmi.edu )

Johns Hopkins University School of Medicine https://orcid.org/0000-0002-5196-7647

Research article

Keywords: electronic medical records, stigma, multiple sclerosis, societal stigma, self stigma 
Posted Date: March 9th, 2020

DOl: https://doi.org/10.21203/rs.3.rs-16332/v1

License: (c) (i) This work is licensed under a Creative Commons Attribution 4.0 International License. Read Full License

Version of Record: A version of this preprint was published at Journal of Affective Disorders on March 1st, 2021. See the published version at https://doi.org/10.1016/j.jad.2020.12.167. 


\section{Abstract}

Background: Electronic medical records (EMRs) have transformed the way healthcare professionals manage and share patient data while providing integrated and comprehensive care. However, the rate of EMR use among psychiatrists is generally lower compared to physicians in other medical disciplines, in part due to concerns over patients' experience of stigma surrounding mental health. This paper explores the willingness to share medical records among people with multiple sclerosis (MS), who experience higher rates of psychiatric comorbidities compared to the general population. It also examines the role that stigma plays in patients' preferences regarding the sharing of their electronic medical records. Methods: MS patients were surveyed regarding their co-occurring psychiatric and non-psychiatric diagnoses, willingness to share their health information electronically among their treating doctors, and levels of self and societal stigma associated with their various co-occurring diagnoses. Results: $96.44 \%$ and $87.14 \%$ of participants were willing to share their non-psychiatric and psychiatric diagnoses, respectively; $97.70 \%$ and $92.78 \%$ were willing to share non-psychiatric and psychiatric medications, respectively. MS patients with a psychiatric co-occurring diagnosis, compared to those without, were significantly more likely to share their psychiatric diagnosis $(A O R=2.59)$ and psychiatric medications $(A O R=3.19)$. Those with both non-psychiatric and psychiatric co-occurring diagnoses were significantly more likely to share their psychiatric diagnosis $(A O R=3.84)$ and psychiatric medications $(A O R=7.02)$ than patients with no co-occurring diagnosis other than MS. Five (substance use, personality, eating, psychotic, and neurodevelopmental disorders) of the top six diagnoses for which societal stigma was greater than self stigma, and three (sexual, anxiety, and mood disorders) of the top five diagnoses for which self stigma was greater than societal stigma were psychiatric diagnoses. High levels of societal stigma correlated with decreased likelihood in sharing non-psychiatric medications, while high levels of self stigma were associated with a greater decrease in patient willingness to share psychiatric medications. Conclusions: Despite the presence of stigma decreasing patient willingness to share medical records, people with MS who had psychiatric disorders, compared to those without, endorsed greater willingness to share their health records electronically.

\section{Background}

Over the past decade, most hospitals have replaced paper-based medical records with electronic health charts. An electronic medical record (EMR) is a computer-based storehouse of a patient's medical information: it contains a patient's health problems, health professionals' notes, laboratory results, prescribed medications, treatment plans, and other relevant health information [1]. Research suggests that the use of EMRs can improve patient care by increasing accuracy, communicating information rapidly, and facilitating care coordination among a patient's multiple care providers [1-6]. Given these benefits, governmental support initiatives, most notably the American Recovery and Reinvestment Act of 2009 , have allocated billions of dollars in funds to encourage hospitals to make the transition to EMR systems [1]. 
Despite these incentives, many psychiatric departments are reluctant to embrace electronic medical records [7-10]. Psychiatrists are significantly less likely to use EMRs compared to other physicians [11], and they are the least likely among all medical specialties to apply for governmental EMR incentives [12]. The low rate of EMR use among psychiatrists is partly due to increased concern about the privacy and security of psychiatric information, which is considered to be highly sensitive because psychiatric disorders are associated with high levels of stigma [13-16]. Consequently, psychiatric information is allotted special protections in an attempt by psychiatrists to insulate their patients from the effects of stigma and discrimination $[5,17,18]$.

Despite concerns that the incorporation of psychiatric information into EMRs may negatively impact psychiatric patients' privacy, research suggests that psychiatric EMRs improve patients' mental health care and health outcomes. In one study, inclusion of depression screening information in an EMR resulted in increased treatment of depression and follow-up care by a primary care physician [19]. Similar results have been found for the screening and treatment of bipolar disorder [20]. In another study, hospitals that utilized electronic psychiatric records and made them accessible to other non-psychiatric treating physicians reported lower readmission rates among psychiatric patients [2].

Dissemination of a patient's psychiatric health information to all of their treating physicians may be particularly important for those patients suffering from non-psychiatric illnesses that have high rates of psychiatric comorbidities, such as multiple sclerosis (MS). MS is a chronic, neuroinflammatory disease that carries a high rate of clinical depression, with lifetime risk estimated at 50\% [21, 22]. Because the course of MS and depression are often related, it is critical for both their medical and psychiatric care to be integrated and coordinated [23].

There is a dearth of research conducted on the attitudes of people with MS toward sharing psychiatric information electronically. Prior research in other patient populations has established that, although there are differences among patients' willingness to share "sensitive" vs. "non-sensitive" medical information, the majority of patients are willing to have their sensitive health information shared through EMR systems [24]. In one study, $70 \%$ of patients were willing to share sensitive information with their doctors and nurses, and $78 \%$ of patients were willing to share non-sensitive medical information [25]. Caine and Hanania similarly found that, among a sample of patients whose medical records contained sensitive information, $95 \%$ were willing to share "less-sensitive" information, and $76 \%$ were willing to share "highly sensitive" information [26]. In one of the few studies specifically addressing psychiatric information, only $5 \%$ of patients surveyed declined to share their psychiatric records electronically [27]. An understanding of patient willingness to share information of varying sensitivity is a good start to understanding the long-term acceptability and feasibility of EMRs, and further studies focusing on experiences of stigma can elucidate these patterns in EMR sharing.

To our knowledge, no study to date has examined whether experiences of health-related stigma account for patients' attitudes toward sharing of their health information in EMRs. Given that people with MS can benefit greatly from comprehensive, multidisciplinary care-including collaborative psychiatric care-the 
objective of the present study was to clarify attitudes toward EMR sharing of psychiatric information by people with MS, and how these attitudes relate to experiences of stigma. The first aim of this study was to compare MS patients with and without co-occurring psychiatric diagnoses in terms of their willingness to share their diagnoses and medications through EMRs. The second aim of the study was to examine the association between patients' experiences of stigma (both self-stigma and societal stigma) and their willingness to share their diagnoses and medications through EMRs. We hypothesized that the majority of participants would be willing to share both medical and psychiatric information, but that those patients who had first-hand knowledge of having a psychiatric illness would be more willing to share their psychiatric records than patients without a psychiatric diagnosis. The implications of this research to inform health care providers about the preferences of their patient regarding sharing of their medical and psychiatric health information can help prevent miscommunication that can negatively impact patients' healthcare.

\section{Method}

\section{Sample}

A link to a survey was provided to members of the Multiple Sclerosis Association of America (MSAA) via an online email listserv. The MSAA is a not-for-profit organization that aims to educate the public about Multiple Sclerosis and provide resources to people with MS and their families. Subscribers of MSAA's email update listserv received an email that invited them to "participate in a research study survey to examine patients' opinions on physician use of electronic medical records." Of the 3716 who consented to participate, 3020 participants endorsed having MS and were included in the data analysis. Participants who indicated that they did not have a neurological diagnosis $(n=242)$, or who did not answer $(n=454)$, were excluded from analysis. Sample demographics are shown in Table 1.

\section{Measures}

Participants were asked to report if they currently have any of the following diagnoses: mood disorder, eating disorder, anxiety disorder, neurodevelopmental disorder, psychotic disorder, personality disorder, sexual disorder, and substance use disorder. They were also asked if they add additional diagnoses of cancer, digestive disorder, reproductive cancer, neurological disorder, endocrine disorder, cardiovascular disorder, genitourinary disorder, liver disorder, respiratory disorder, vision disorder, sexual transmitted infection, skin disorder, or infectious disease. The former 8 diagnosis categories were labeled "psychiatric" diagnoses, and the latter 13 diagnosis categories were labeled "non-psychiatric" diagnoses. In order to assess attitudes toward sharing different types of health information, all participants were also asked to indicate, if available, whether they would be willing to share records that included information related to their: psychiatric diagnoses, non-psychiatric medical diagnoses, psychiatric medications, and non-psychiatric medications.

Based on these categories, MS patient participants were separated into 4 groups: those with no reported co-occurring diagnosis $(n=929)$; those with only a non-psychiatric co-occurring diagnosis $(n=718)$; those 
with only a psychiatric co-occurring diagnosis $(n=558)$; and those with both co-occurring non-psychiatric and psychiatric diagnoses $(n=818)$. For each diagnosis reported, participants were asked to rate the level of societal stigma and self-stigma on a Likert-type scale, ranging from 1 ("no stigma") to 10 ("high stigma"). Societal stigma was defined as "negative attitudes and/or discrimination from others." Selfstigma was defined as "negative feelings you have about yourself and your illness." For each study participant, the highest score of societal stigma and self-stigma for psychiatric diagnosis and nonpsychiatric diagnosis was selected. The categorical stigma variable is defined as follows: no stigma (rating of 1); moderate stigma (ratings of 2-5); and high stigma (ratings of 6-10).

\section{Statistical Analyses}

Given that the rate of missing responses was $<10 \%$ for each item, data were not imputed to account for these missing values. A multivariable logistic regression was used for each outcome variable: willingness to share psychiatric diagnosis, willingness to share non-psychiatric diagnosis, willingness to share psychiatric medications, and willingness to share non-psychiatric medications. The main explanatory variable was stigma, which was split into four types: non-psychiatric self-stigma, non-psychiatric societal stigma, psychiatric self-stigma, and psychiatric societal stigma. Results were expressed using adjusted odds ratio (AOR). The "stigma type differential," which describes the difference in self-stigma and societal stigma for each diagnosis, was calculated by subtracting the average reported societal stigma from the average self stigma for each diagnosis. In each regression analysis, the following variables were adjusted as covariates: diagnosis category, age, gender, race, ethnicity, education level, marital status, and employment level. A cutoff level of $p<.05$ was used to determine statistical significance. Results were analyzed using STATA v.14.

\section{Results}

Overall, $96.44 \%$ of participants were willing to share their non-psychiatric diagnosis and $87.14 \%$ were willing to share their psychiatric diagnosis; $97.70 \%$ were willing to share their non-psychiatric medications and $92.78 \%$ were willing to share their psychiatric medications. These were generally higher than previously reported $[25,26]$, and also suggest patients may be somewhat more willing to share their medications than diagnoses.

Regression analysis showed that most demographic factors (age, gender, marital status, employment, ethnicity, and race) did not have statistically significant effects on likelihood of sharing psychiatric or non-psychiatic diagnoses and medications. Having higher education ( $>12$ years vs. $<12$ years) consistently increased the odds ratio of sharing all types of health information electronically. Regression results indicated that MS patients with only a co-occurring psychiatric diagnosis were significantly more likely to share their psychiatric diagnosis ( $A O R=2.59)$, psychiatric medications ( $A O R=3.19$ ), and nonpsychiatric medications $(A O R=6.16)$ than patients with no co-occurring diagnosis (Table $2 \&$ Table 3 ). Similarly, patients with both co-occurring psychiatric and non-psychiatric diagnoses were significantly more likely to share their psychiatric diagnosis $(A O R=3.84)$, psychiatric medications $(A O R=7.02)$, nonpsychiatric diagnosis $(A O R=4.22)$, and non-psychiatric medications $(A O R=16.37)$ than patients with no 
co-occurring diagnosis (Table $2 \&$ Table 3 ). Those with both psychiatric and non-psychiatric comorbidities are prescribed medications that could potentially interact with one another, and are therefore more likely to disclose their diagnoses and medications, which is reflected in the high AORs in this patient population.

When asked "Which of the following type(s) of information, if available, do you feel are important for a doctor to have in order to provide the best medical care possible?" most participants indicated they believe it was important to share non-psychiatric diagnoses and medications with their doctors. The percentage of participants who believe sharing their psychiatric diagnosis was important for receiving better medical care is greater among patients with co-occurring psychiatric diagnoses: $37.59 \%$ among those with no co-occurring diagnosis, $42.82 \%$ for those with only a non-psychiatric diagnosis, $58.95 \%$ for those with only a psychiatric co-occurring diagnosis, and $67.64 \%$ for those with both types of diagnoses. The same was true for beliefs regarding sharing psychiatric medications: $38.45 \%$ among those with no co-occurring diagnosis, $44.07 \%$ for those with only a non-psychiatric diagnosis, $64.92 \%$ for those with only a psychiatric diagnosis, and $74.73 \%$ for those with both types of diagnoses (Table 5 ). These results offer the explanation that patients with co-occurring psychiatric diagnoses are more likely to share psychiatric health information with their doctors because they believe it is important in order to receive the best care possible.

The average reported self and societal stigmas and calculated stigma type differentials, which have a negative value for diagnoses that have a greater average societal stigma than self stigma and vice versa for positive values, are shown in Fig. 1. Psychiatric diagnoses tended to rank the highest for diagnoses that had the greatest difference in reported self vs. societal stigmas. Substance use, personality, eating, psychotic, and neurodevelopmental disorders were among the six diagnoses with most negative stigma type differential, and sexual, anxiety, and mood disorders were among the five diagnoses with most positive stigma type differentials. Stigma type differentials and likelihood of sharing medical records electronically for each diagnosis are summarized in Table 5.

Regression analyses indicated that after adjusting for age, gender, education, marital status, employment, race, and diagnosis category, patients who reported moderate levels of psychiatric self-stigma were less willing to share their psychiatric medications (AOR 0.39) and psychiatric diagnosis (AOR $=0.47)$ than were patients who reported no psychiatric self-stigma (Table 4). Patients who reported high levels of nonpsychiatric societal stigma were significantly less likely to share their non-psychiatric medication records $(A O R=0.20)$ than were patients who reported no non-psychiatric societal stigma. Having a high level of perceived non-psychiatric societal stigma significantly decreased likelihood of sharing non-psychiatric medications, while a high level of psychiatric societal stigma did not decrease likelihood of sharing psychiatric medications. Conversely, high levels of psychiatric self stigma decreased likelihood of sharing psychiatric medications, while high levels of non-psychiatric self stigma did not result in a decrease in sharing non-psychiatric medications. These results suggest that self and societal stigma differentially affect willingness toward sharing psychiatric vs. psychiatric medications. 


\section{Discussion}

The current study was designed to investigate patients' attitudes toward sharing non-psychiatric and psychiatric health information with physicians through EMRs. Findings from our study indicate that most people in the sample were willing to share both psychiatric and non-psychiatric information, and that the difference in sharing psychiatric vs. non-psychiatric medical information is smaller than previously reported or commonly expected $[26,28]$. This indicates that although hospitals and physicians commonly categorize psychiatric health information as "sensitive," and are less likely to share psychiatric diagnoses and medications with other care providers, patients themselves would be more willing for this information to be shared because of their perceived benefits on their healthcare. In regression analyses, having a psychiatric diagnosis was significantly associated with willingness to share information electronically, despite stigma. This finding suggests that patients who have a psychiatric disorder may actually endorse higher willingness to share psychiatric information because perhaps they understand the importance of EMRs as a means to ensure their multi-disciplinary health care team can provide an integrated and coherent approach to the care they receive. It is also possible that having and receiving treatment for a psychiatric diagnosis decreases the associated stigma, contributing to the increased likelihood of sharing psychiatric health information.

Results also highlighted the importance of stigma in willingness to share health information through EMRs. As expected, higher rates of both self- and societal stigma significantly correlated with a decreased likelihood of participants wishing to share their medical records. Additionally, results indicating that societal stigma seems to more strongly discourage patients from sharing non-psychiatric medications, while self stigma more so discourages sharing of psychiatric mediations. These findings contribute to our understanding of how these two types of stigma differentially affect different types of conditions. High stigma and sensitivity surrounding mental health information is often cited as the reason for not sharing psychiatric information [28]; however, these decisions are usually based on assumptions by clinicians or administrators about patient's hesitancy in sharing their own psychiatric health information, rather than the patient's own wishes. Moreover, whereas patient's with non-psychiatric illnesses are rarely if ever asked if their sensitive health information should be included in their EMR's, and psychiatric patient have their health information excluded often without inquiring their preferences. This creates a double standard that largely affects patients who associate high levels of stigma with their non-psychiatric conditions and still have their health records shared because information regarding certain non-psychiatric conditions are not commonly considered "sensitive." Not only does this perpetuate the stigma surrounding mental illness, but it may result in "protecting" patients with psychiatric illnesses from getting optimal care.

Interestingly, patients' report that substance use, personality, and eating disorders have the largest difference in societal > self stigma might better inform our understanding of patient perception of their own illness, their approach to seeking help, and approaches to what motivates them to engage in care. Reporting a higher level of societal stigma compared to self-stigma indicates that these patients perhaps perceive their own illness with less severity or harbor fewer negative feelings toward themselves or their 
illness compared to how they believe others feel about their diagnosis. For example, one of the diagnostic features of anorexia nervosa is a "disturbance in the way in which one's body weight or shape is experienced... or persistent lack of recognition of the seriousness of the current low body weight," suggesting that they are relatively free of self-stigma and feel afflicted by societal stigma. It is interesting to speculate whether patients with substance use disorders, who may suffer from a similar lack of recognition of the seriousness of their drug use behavior, may feel more motivated to avoid societal stigma than driven to change their behavior based on personal negative feelings about their substance use. These feelings may then hinder patients from recognizing that they need help or increase reluctance to seek treatment.

Limitations to this study include the use of single items to measure stigma instead of standardized scales. There is also a potential lack of generalizability from MS patients to patients with psychiatric and non-psychiatric diseases without MS. The patients were engaged in completing the survey based on it being advertised as an opportunity to describe their feelings about electronic health records, without any reference to psychiatric or non-psychiatric differences. Thus, we doubt there was a selection bias regarding differences in patients' feelings about psychiatric vs non-psychiatric co-occurring illnesses. It would be useful for future research to investigate more representative samples in order to gain a better understanding of how willing those with non-psychiatric and psychiatric co-diagnoses are to share medical records and comparing the self- and societal stigma among these two groups. Further studies may also elucidate how these two categories of stigma differentially contribute to patients' and physicians' willingness to use EMRs and how interventions may serve to decrease stigma in order to better patient care.

\section{Conclusions}

In conclusion, this study has shown that a higher proportion of people with MS are willing to share their psychiatric health information in EMR's compared to previously reported rates. It also provides insight into patient perspectives on stigma attributed to psychiatric and non-psychiatric illnesses. These findings suggest that having a psychiatric comorbidity actually increases willingness to share medical records electronically, despite stigma. This is important to take into account as we push forth in incorporating technology in healthcare in order to improve coordination of care for patients with psychiatric and nonpsychiatric comorbidities.

\section{List Of Abbreviations}

$\mathrm{EMR}=$ electronic medical record

MS = multiple sclerosis

MSAA = Multiple Sclerosis Association of America

$\mathrm{AOR}=$ adjusted odds ratio 


\section{Declarations}

Ethics approval and consent to participate

This study was approved by the Johns Hopkins Institutional Review Board. The first page of the survey was a consent form and all participants must have provided written consented to participate before viewing and submitting the survey.

Consent for publication

$\mathrm{N} / \mathrm{A}$

Availability of data and materials

The datasets used and/or analyzed during the current study are available from the corresponding author on reasonable request.

Competing interests

The authors declare that they have no competing interests.

Funding

Philanthropic funding from the Downeys and Mulligans Fore MS provided salary support for research staff. No commercial funding sources were used.

\section{Authors' contributions}

Adam Kaplin will be designated as AK1 and Anupama Kumar as AK2. AA, AM, MP, KJ, and AK1 contributed to the study design. EY, AA, AR, CF, SA, DT, and AK1 contributed to data collection and analysis. RS provided statistical support. EY, AA, and AK1 drafted the manuscript. AH, AM, and AK2 provided substantive input and revisions. All authors have read and approved the manuscript.

Acknowledgements

$\mathrm{N} / \mathrm{A}$

\section{References}

1. Plovnick RM. The Progression of Electronic Health Records and Implications for Psychiatry. Am J Psychiatry. 2010 May;167(5):498-500. doi: 10.1176/appi.ajp.2009.09101473

2. Kozubal DE, Samus QM, Bakare AA, Trecker CC, Wong H-W, Guo H, et al. Separate may not be equal: A preliminary investigation of clinical correlates of electronic psychiatric record accessibility in academic medical centers. Int J Med Inform. 2013 Apr;82(4):260-7. doi:

10.1016/j.ijmedinf.2012.11.007

Page 10/17 
3. Chaudhry B, Wang J, Wu S, Maglione M, Mojica W, Roth E, et al. Systematic review: impact of health information technology on quality, efficiency, and costs of medical care. Ann Intern Med. 2006 May 16;144(10):742-52.

4. Hillestad R, Bigelow J, Bower A, Girosi F, Meili R, Scoville R, et al. Can Electronic Medical Record Systems Transform Health Care? Potential Health Benefits, Savings, And Costs. Health Aff. 2005 Sep;24(5):1103-17. doi: 10.1377/hlthaff.24.5.1103

5. Triplett P. Psychiatry and the Meaningful Use of Electronic Health Records. Perspect Biol Med. 2013;56(3):407-21. doi: 10.1353/pbm.2013.0028

6. Tsai J, Bond G. A comparison of electronic records to paper records in mental health centers. Int J Qual Heal Care. 2007 Dec 11;20(2):136-43. doi: 10.1093/intqhc/mzm064

7. Clemens NA. Privacy, Consent, and the Electronic Mental Health Record. J Psychiatr Pract. 2012 Jan;18(1):46-50. doi: 10.1097/01.pra.0000410987.38723.47

8. Ennis L, Rose D, Callard F, Denis M, Wykes T. Rapid progress or lengthy process? electronic personal health records in mental health. BMC Psychiatry. 2011 Dec 26;11(1):117. doi: 10.1186/1471-244X11-117

9. Freedman J. The role of information technology in evidenced-based practice. Psychiatr Clin North Am. 2003 Dec;26(4):833-50, vii-viii.

10. Khalifa M. Barriers to Health Information Systems and Electronic Medical Records Implementation. A Field Study of Saudi Arabian Hospitals. Procedia Comput Sci. 2013 Jan 1;21:335-42. doi: 10.1016/J.PROCS.2013.09.044

11. Kokkonen EWJ, Davis SA, Lin H-C, Dabade TS, Feldman SR, Fleischer AB. Use of electronic medical records differs by specialty and office settings. J Am Med Informatics Assoc. 2013 Jun 1;20(e1):e33-8. doi: 10.1136/amiajnl-2012-001609

12. Hsiao C-J, Decker SL, Hing E, Sisk JE. Most physicians were eligible for federal incentives in 2011, but few had EHR systems that met meaningful-use criteria. Health Aff (Millwood). 2012 May;31(5):1100-7. doi: 10.1377/hlthaff.2011.1315

13. Chew-Graham CA, Rogers A, Yassin N. "I wouldn't want it on my CV or their records": medical students' experiences of help-seeking for mental health problems. Med Educ. 2003 Oct;37(10):87380. doi: 10.1046/j.1365-2923.2003.01627.x

14. Clement S, Schauman O, Graham T, Maggioni F, Evans-Lacko S, Bezborodovs N, et al. What is the impact of mental health-related stigma on help-seeking? A systematic review of quantitative and qualitative studies. Psychol Med. 2015 Jan 21;45(1):11-27. doi: 10.1017/S0033291714000129

15. Eisenberg D, Downs MF, Golberstein E, Zivin K. Stigma and Help Seeking for Mental Health Among College Students. Med Care Res Rev. 2009 Oct 19;66(5):522-41. doi: 10.1177/1077558709335173

16. Sartorius N. Stigma and mental health. Lancet. 2007 Sep 8;370(9590):810-1. doi: 10.1016/S01406736(07)61245-8

17. Greene J. Behavioral Health Data in the Electronic Health Record. Ann Emerg Med. 2013 Oct;62(4):A19-21. doi: 10.1016/j.annemergmed.2013.08.003 
18. Stewart C, Spicer M, Babl FE. Caring for adolescents with mental health problems: challenges in the emergency department. J Paediatr Child Health. 2006 Nov;42(11):726-30. doi: 10.1111/j.14401754.2006.00959.x

19. Gill JM, Dansky BS. Use of an Electronic Medical Record to Facilitate Screening for Depression in Primary Care. Prim Care Companion J Clin Psychiatry. 2003 Jun;5(3):125-8.

20. Gill JM, Chen YX, Grimes A, Klinkman MS. Using Electronic Health Record-Based Tools To Screen for Bipolar Disorder in Primary Care Patients With Depression. J Am Board Fam Med. 2012 May 1;25(3):283-90. doi: 10.3122/jabfm.2012.03.110217

21. Feinstein A. Multiple sclerosis and depression. Mult Scler J. 2011 Nov 4;17(11):1276-81. doi: $10.1177 / 1352458511417835$

22. Mohr D. Treatment of depression in multiple sclerosis: review and meta-analysis. Clin Psychol Sci Pract. 1999 Feb 1;6(1):1-9. doi: 10.1093/clipsy/6.1.1

23. Stewart RF, Kroth PJ, Schuyler M, Bailey R. Do electronic health records affect the patient-psychiatrist relationship? A before \&amp; after study of psychiatric outpatients. BMC Psychiatry. $2010 \mathrm{Dec}$ 8;10(1):3. doi: 10.1186/1471-244X-10-3

24. Schwartz PH, Caine K, Alpert SA, Meslin EM, Carroll AE, Tierney WM. Patient preferences in controlling access to their electronic health records: a prospective cohort study in primary care. J Gen Intern Med. 2015 Jan;30 Suppl 1(Suppl 1):S25-30. doi: 10.1007/s11606-014-3054-z

25. Whiddett R, Hunter I, Engelbrecht J, Handy J. Patients' attitudes towards sharing their health information. Int J Med Inform. 2006 Jul 1;75(7):530-41. doi: 10.1016/J.IJMEDINF.2005.08.009

26. Caine K, Hanania R. Patients want granular privacy control over health information in electronic medical records. J Am Med Inform Assoc. 2013 Jan 1;20(1):7-15. doi: 10.1136/amiajnl-2012001023

27. Flynn HA, Marcus SM, Kerber K, Alessi N. Patients' Concerns About and Perceptions of Electronic Psychiatric Records. Psychiatr Serv. 2003 Nov;54(11):1539-41. doi: 10.1176/appi.ps.54.11.1539

28. Salomon RM, Blackford JU, Rosenbloom ST, Seidel S, Clayton EW, Dilts DM, et al. Openness of patients' reporting with use of electronic records: psychiatric clinicians' views. J Am Med Informatics Assoc. 2010 Jan 1;17(1):54-60. doi: 10.1197/jamia.M3341

\section{Tables}

Table 1

Demographic Characteristics of Sample $(N=3023)$. 


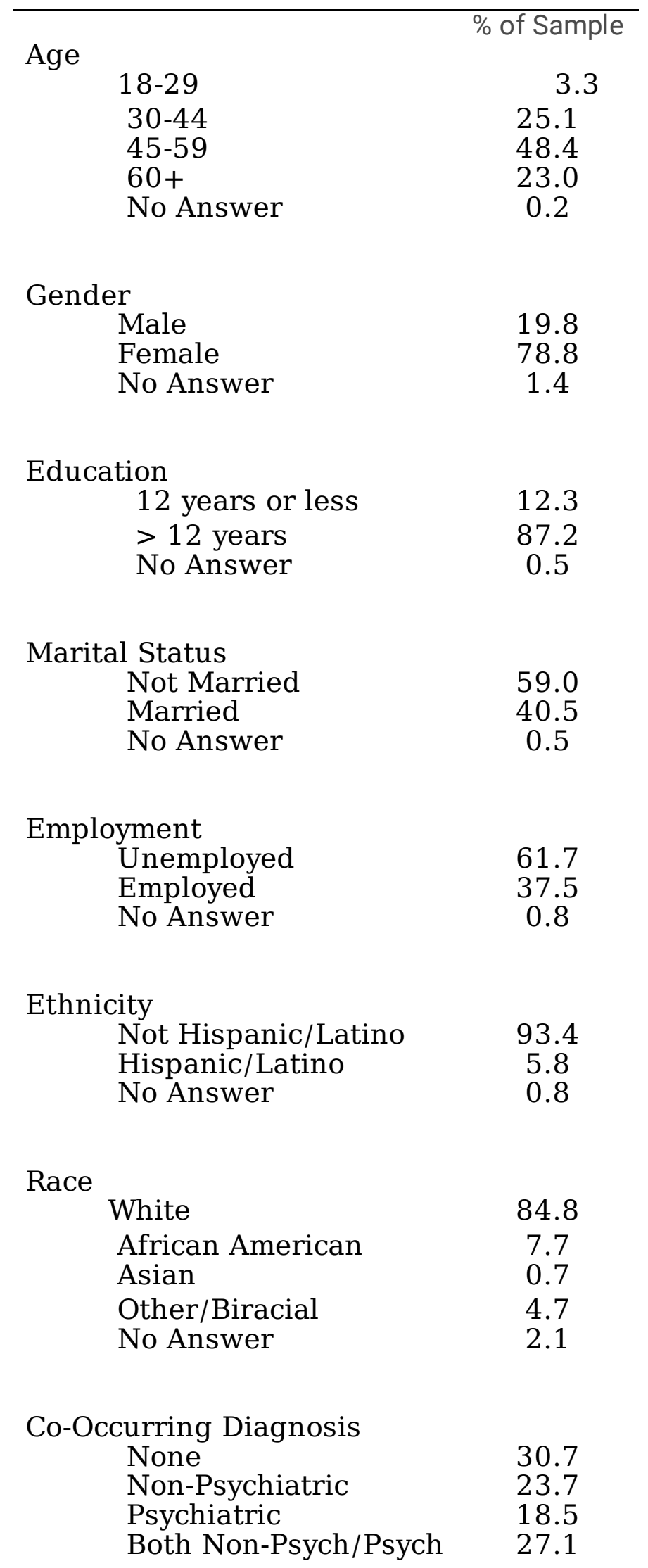

Table 2 
Logistic multiple regression analysis of willingness to share psychiatric and non-psychiatric diagnoses and medications

\begin{tabular}{|c|c|c|c|c|c|c|c|c|}
\hline \multirow{2}{*}{ Co-Occurring Diagnosis $\mathrm{C}$} & \multicolumn{2}{|c|}{ Psychiatric Diagnosis } & \multicolumn{2}{|c|}{ Non-Psychiatric Diagnosis } & \multicolumn{2}{|c|}{ Psychiatric Medications } & \multicolumn{2}{|c|}{ Non-psychiatric Medications } \\
\hline & $\mathrm{AOR}^{\mathrm{a}}$ & $\mathrm{CI}^{\mathrm{b}}$ & $\mathrm{AOR}^{\mathrm{a}}$ & $\mathrm{CI}^{\mathrm{b}}$ & $\mathrm{AOR}^{\mathrm{a}}$ & $\mathrm{CI}^{\mathrm{b}}$ & $\mathrm{AOR}^{\mathrm{a}}$ & $\mathrm{CI}^{\mathrm{b}}$ \\
\hline None & 1.00 & -- & 1.00 & --- & 1.00 & -- & 1.00 & ---- \\
\hline Non-Psychiatric & 1.14 & $0.82-1.60$ & 1.52 & $0.79-2.93$ & $1.90^{* *}$ & $1.18-3.04$ & 2.33 & $0.93-5.86$ \\
\hline Psychiatric & $2.59 * *$ & $1.46-4.59$ & 2.26 & $0.80-6.16$ & $3.19^{* *}$ & $1.47-6.91$ & $6.16^{* *}$ & $1.70-22.33$ \\
\hline Both Non-Psych/Psych & $3.84 * *$ & $2.14-6.88$ & $4.22 * *$ & $1.43-12.47$ & $7.02 * *$ & $3.09-15.96$ & $16.37^{* *}$ & $3.82-70.11$ \\
\hline
\end{tabular}

${ }^{a}=$ Adjusted Odds Ratio, ${ }^{b}=$ Confidence Interval, ${ }^{c}=$ Type of diagnosis co-occurring with $M S, * p<.05, * * p<.01$

Table 3

Effects of self and societal stigma on willingness to share psychiatric and non-psychiatric diagnosis. 


\begin{tabular}{|c|c|c|c|c|}
\hline \multirow[t]{2}{*}{ Covariates } & \multicolumn{2}{|c|}{ Psychiatric Diagnosis } & \multicolumn{2}{|c|}{ Non-Psychiatric Diagnosis } \\
\hline & $\mathrm{AOR}^{\mathrm{a}}$ & $\mathrm{CI}^{\mathrm{b}}$ & $\mathrm{AOR}^{\mathrm{a}}$ & $\mathrm{CI}^{\mathrm{b}}$ \\
\hline \multicolumn{5}{|l|}{ Non-Psychiatric Self Stigma } \\
\hline No Stigma & 1.00 & -- & 1.00 & -- \\
\hline Moderate Stigma & 0.88 & $0.52-1.49$ & 0.67 & $0.24-1.87$ \\
\hline High Stigma & 1.05 & $0.54-2.07$ & 0.50 & $0.15-1.61$ \\
\hline \multicolumn{5}{|l|}{ Non-Psychiatric Soc Stigma } \\
\hline No Stigma & 1.00 & -- & 1.00 & -- \\
\hline Moderate Stigma & 0.99 & $0.58-1.71$ & 0.94 & $0.34-2.64$ \\
\hline High Stigma & 0.61 & $0.31-1.19$ & 0.70 & $0.22-2.25$ \\
\hline \multicolumn{5}{|l|}{ Psych Self Stigma } \\
\hline No Stigma & 1.00 & -- & 1.00 & -- \\
\hline Moderate Stigma & $0.47 *$ & $0.25-0.89$ & 0.42 & $0.13-1.35$ \\
\hline High Stigma & 0.75 & $0.37-1.52$ & 0.60 & $0.17-2.16$ \\
\hline \multicolumn{5}{|l|}{ Psych Soc Stigma } \\
\hline No Stigma & 1.00 & -- & 1.00 & -- \\
\hline Moderate Stigma & 0.86 & $0.49-1.50$ & 1.44 & $0.52-3.99$ \\
\hline High Stigma & 0.76 & $0.41-1.42$ & 0.79 & $0.27-2.32$ \\
\hline
\end{tabular}

${ }^{a}=$ Adjusted Odds Ratio, ${ }^{b}=$ Confidence Interval, Soc stigma = "negative attitudes and/or discrimination from others" Self-stigma = "negative feelings you have about yourself and your illness, ${ }^{*} p<.05, * * p<.01$

Table 4

Effects of self and societal stigma on willingness to share psychiatric and non-psychiatric medications 


\begin{tabular}{|c|c|c|c|c|}
\hline \multirow[b]{2}{*}{ Covariates } & \multicolumn{2}{|c|}{ Psychiatric Medications } & \multicolumn{2}{|c|}{ Non-psychiatric Medications } \\
\hline & $\mathrm{AOR}^{\mathrm{a}}$ & $\mathrm{CI}^{\mathrm{b}}$ & $\mathrm{AOR}^{\mathrm{a}}$ & $\mathrm{CI}^{\mathrm{b}}$ \\
\hline \multicolumn{5}{|l|}{ Non-Psych Self Stigma } \\
\hline No Stigma & 1.00 & -- & 1.00 & -- \\
\hline Moderate Stigma & 0.64 & $0.31-1.35$ & 0.86 & $0.22-3.30$ \\
\hline High Stigma & 0.73 & $0.29-1.84$ & 1.91 & $0.36-10.11$ \\
\hline \multicolumn{5}{|l|}{ Non-Psych Soc Stigma } \\
\hline No Stigma & 1.00 & -- & 1.00 & -- \\
\hline Moderate Stigma & 0.88 & $0.41-1.87$ & 0.39 & $0.10-1.58$ \\
\hline High Stigma & 0.49 & $0.20-1.21$ & $0.20 *$ & $0.04-0.97$ \\
\hline \multicolumn{5}{|l|}{ Psych Self Stigma } \\
\hline No Stigma & 1.00 & -- & 1.00 & -- \\
\hline Moderate Stigma & $0.39 *$ & $0.16-0.95$ & 0.59 & $0.11-3.32$ \\
\hline High Stigma & 0.48 & $0.18-1.29$ & 0.94 & $0.15-6.04$ \\
\hline \multicolumn{5}{|l|}{ Psych Soc Stigma } \\
\hline No Stigma & 1.00 & -- & 1.00 & -- \\
\hline Moderate Stigma & 1.05 & $0.50-2.26$ & 0.30 & $0.05-1.71$ \\
\hline High Stigma & 1.23 & $0.51-2.94$ & 0.21 & $0.03-1.32$ \\
\hline
\end{tabular}

${ }^{a}=$ Adjusted Odds Ratio, ${ }^{b}=$ Confidence Interval, ${ }^{*} p<.05,{ }^{* *} p<.01$

\section{Table 5}

Percent of patients who believe sharing different types of health information will benefit their medical care 


\begin{tabular}{|c|c|c|c|}
\hline Co-Occurring Diagnosis & Sharing Psychiatric & Sharing Non-Psychiatric & Sharing Psychiatric \\
& Diagnosis & Diagnosis & Medications \\
\hline None & $37.59 \%$ & $89.37 \%$ & $38.45 \%$ \\
\hline Non-Psychiatric & $42.82 \%$ & $92.19 \%$ & $44.07 \%$ \\
\hline Psychiatric & $58.95 \%$ & $91.14 \%$ & $64.92 \%$ \\
\hline Both Non- & $67.64 \%$ & $94.99 \%$ & $74.73 \%$ \\
\hline
\end{tabular}

${ }^{c}=$ Type of diagnosis co-occurring with $M S$

\section{Figures}

A)

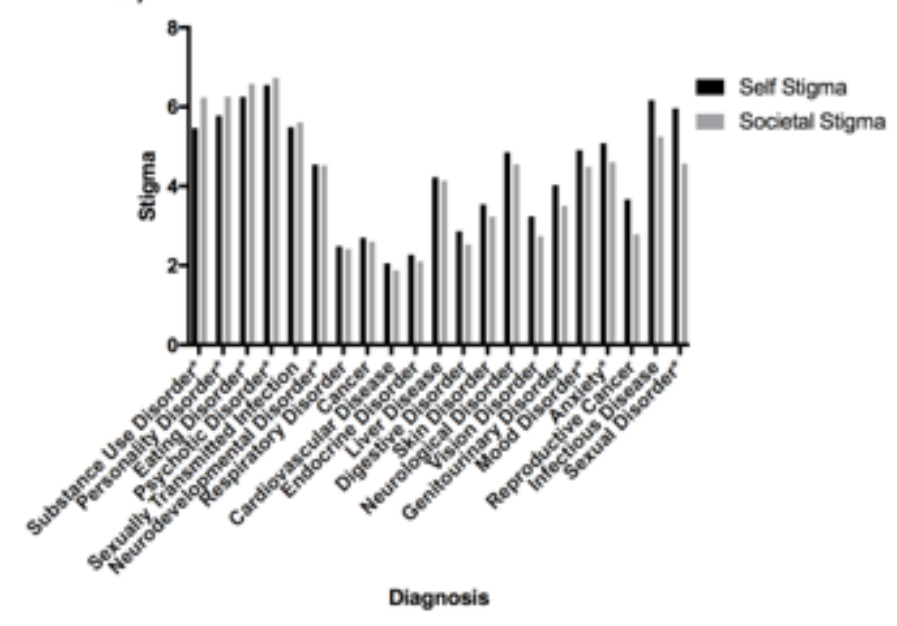

B)

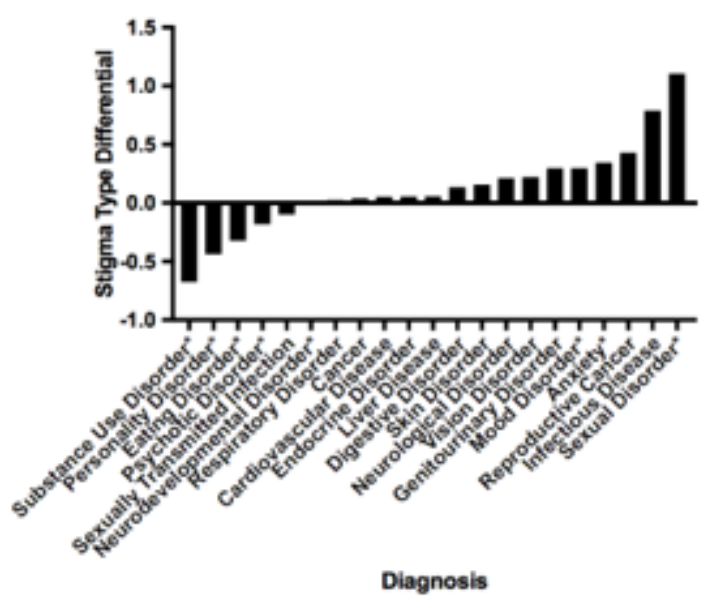

\section{Figure 1}

A) Average self and societal stigma reported by participants with each diagnosis B) Difference in average self and societal stigmas, weighted by average overall stigma for that diagnosis. *Indicates psychiatric diagnosis; negative values indicate societal stigma is greater than self stigma

\section{Supplementary Files}

This is a list of supplementary files associated with this preprint. Click to download.

- SupplementalTable.docx 